\title{
Staphylococcus aureus resistentes a meticilina (SARM) positivos para PVL aislados en individuos sanos de Montería-Córdoba
}

\author{
Dayan Lozano, Loly Díaz, Marcela Echeverry, Sara Pineda, Salim Máttar* \\ Universidad de Córdoba. Instituto de Investigaciones Biológicas del Trópico (IIBT), Facultad de Medicina Veterinaria y Zootecnia, \\ Montería, Colombia. http://www.unicordoba.edu.co/institutos/iibt/ \\ *mattarsalim@hotmail.com
}

Recibido: 11-11-2009; Aceptado: 09-06-2010

\begin{abstract}
Resumen
Objetivo. Estudiar cepas asociadas a la comunidad de SARM y productoras de PVL en individuos sanos de la ciudad de Montería. Materiales y métodos. Estudio descriptivo, prospectivo de corte transversal, a partir de un total de 253 muestras obtenidas de hisopados faríngeos en tres comunidades: 91 internos de la cárcel de Montería (19-58 años), 100 estudiantes adultos jóvenes de la Universidad de Córdoba (17-30 años) y 62 niños en edad escolar (4-9 años) del Colegio de la Universidad de Córdoba. Los individuos participantes no estuvieron hospitalizados en los últimos meses, ni recibieron tratamiento antimicrobiano y no presentaron signos y/o síntomas clínicos. Los aislamientos fueron identificados por pruebas convencionales microbiológicas y se determinó la susceptibilidad antimicrobiana para los diferentes antibióticos a través de MicroScan ${ }^{\mathrm{TM}}$ combo $1 \mathrm{~A}$ (SIEMENS). Para la detección de los genes $n u c, m e c A$ y PVL se utilizaron los protocolos de Brakstad et al (5), Oliveira et al (6) y Gerard et al (7) respectivamente. Resultados. De las 253 muestras analizadas, $62(24,5 \%)$ resultaron positivas para S. aureus, de éstas 4 (6,45\%) fueron resistentes a meticilina, de las cuales $2(25 \%)$ resultaron PVL positivas; 58 (93,54\%) fueron sensibles y 6 (75\%) positivas para PVL. Conclusiones. Los resultados obtenidos demuestran la colonización por SARM-AC en individuos sanos, la colonización fue mayor en la población infantil y adultos jóvenes.
\end{abstract}

Palabras clave: Staphylococcus aureus, resistencia, meticilina, PVL, comunidad, Colombia.

\begin{abstract}
PVL positive methicillin-resistant Staphylococcus aureus (MRSAs) strains isolated from healthy individuals in Montería, Córdoba. Objective. To study SARM and PVL producing strains in healthy individuals of Montería city. Materials and methods. This was a descriptive, prospective and transversal study. A total of 253 samples from pharyngeal swabs in three communities were studied: 91 prisoners of the jail of Montería (19-58 years old), 100 young adult students of the University of Cordoba (17-30 years old) and 62 children in school age (4-9 years old) of the primary school of the University of Cordoba. Individuals analyzed had not been hospitalized, had not received any antimicrobial treatment and did not show any clinical signs or symptoms in the last months. The isolates were identified by conventional microbiological tests and the antimicrobial susceptibility was determined for different antibiotics with MicroScan ${ }^{\mathrm{TM}}$ kit $1 \mathrm{~A}$ (SIEMENS). For the detection of the $n u c, m e c A$ and PVL genes, we followed the protocols of Brakstad et al., Oliveira et al. and Gerard et al. respectively. Results. Of the 253 analyzed samples, $62(24.5 \%)$ were positive for $S$. aureus, out of those $4(6.45 \%)$ were resistant to methicillin and $2(25 \%)$ of them were PVL positive; $58(93.54 \%)$ were sensitive to methicillin and $6(75 \%)$ positive for PVL. Conclusions. Results obtained in this study show the colonization by SARM-AC of healthy individuals. Colonization was higher in young adults and children in school-age.
\end{abstract}

Key words. Staphylococcus aureus, resistance, methicillin, PVL, community, Colombia. 


\section{Resumo}

Staphylococcus aureus resistente à meticilina (SARM) positivos para PVL isolados em indivíduos saudáveis de "Montería, Córdoba". Objetivo. Estudar cepas associada à comunidade de SARM e produtoras de PVL em indivíduos saudáveis da cidade de Monteria. Materiais e métodos. Estudo descriptivo, prospectivo de corte transversal, de um total de 253 amostras obtidas em esfregaços faríngeos em três comunidades: 91 reclusos em Monteria (19-58 anos), 100 alunos adultos jovens da "Universidad de Córdoba" (17 - 30 anos) e 62 crianças em idade escolar (4-9 anos) do "Colégio de la Universidad de Córdoba". Os indivíduos envolvidos não foram internados nos últimos meses, ou receberam tratamento antimicrobiano e não tinham sinais e / ou sintomas clínicos. Os isolados foram identificados por testes microbiológicos convencionais e se determinou a susceptibilidade antimicrobiana aos diferentes antibióticos através de MicroScan TM combo 1A (SIEMENS). Para a detecção de genes nuc, mecA e LPV foram utilizados protocolos Brakstad et al (5), Oliveira et al (6) e Gerard et al (7), respectivamente. Resultados. De 253 amostras testadas, 62 (24,5\%) foram positivas para $S$. aureus, destas 4(6,45\%) foram resistentes à meticilina, dois das quais (25\%) foram LPV positivas, 58 (93,54\%) foram sensíveis e seis (75\%) foram positivas para PVL. Conclusões. Os resultados demonstram a colonização de SARM-AC em indivíduos saudáveis, a colonização foi maior em crianças e adultos jovens.

Palavras-chave: Staphylococcus aureus, resistência, meticilina, PVL, comunidade, Colômbia.

\section{Introducción}

En personas sin contacto reciente con ambientes hospitalarios la presencia de Staphylococcus aureus resistente a meticilina (SARM) puede convertirse en un problema de salud pública. SARM es un patógeno emergente que ha tenido un incremento importante en la prevalencia y ha emergido globalmente en un período corto de tiempo.

Aunque existen diferencias genéticas entre las cepas adquiridas en la comunidad (AC) y las nosocomiales, ambas conservan genes de virulencia comunes como el $m e c A$ y PVL entre otros (1). El factor de virulencia de PantonValentine Leucocidina (PVL) es una citotóxina que se une a los fosfolípidos de la membrana de los leucocitos y macrófagos induciendo la formación de poros que destruyen la célula alterando la permeabilidad celular (2). Se cree que PVL es uno de los factores de virulencia responsable de la alta patogenicidad de estas cepas, lo que ocasiona desde infecciones simples a nivel de piel y tejidos blandos, hasta enfermedades invasivas como neumonía necrotizante, sepsis severa y fascitis necrotizante, que ponen en riesgo la vida del individuo $(1,2)$.

La infección por SARM-AC es un problema particular en niños y adultos jóvenes sanos (3). Los aislamientos de SARM-AC implican un costo epidemiológico y económico, debido al uso de antibióticos para el tratamiento de pacientes ambulatorios, constituyéndose en un problema de salud pública (4). SARM-AC se ha reportado en poblaciones sanas, como atletas, soldados y jóvenes. También desde el punto de vista epidemiológico se complican las diferencias para separar las cepas adquiridas en la comunidad y en el hospital.
El objetivo de éste trabajo fue estudiar cepas asociadas a la comunidad de SARM y productoras de PVL en individuos sanos de la ciudad de Montería.

\section{Materiales y métodos}

\section{Tipo de estudio, localización, población analizada y especimenes}

Se llevó a cabo un estudio de tipo descriptivo prospectivo durante el año 2008. El trabajo se realizó en la ciudad de Montería que tiene una población aproximada de 350.000 habitantes. Se analizaron 253 muestras obtenidas a partir de hisopados faríngeos de individuos sanos pertenecientes a tres comunidades de la siguiente manera: 91 internos de la cárcel nacional las Mercedes de Montería (19-58, años), 100 estudiantes adultos jóvenes (17-30, años) de la Universidad de Córdoba y 62 niños en edad escolar (4-9, años) del Colegio de la Universidad de Córdoba. Los hisopados faríngeos se trasladaron en tubos con $3 \mathrm{ml}$ de agar-agar al $12 \%(\mathrm{p} / \mathrm{v})$ en un tiempo menor a seis horas al Instituto de Investigaciones Biológicas del Trópico (IIBT) de la Universidad de Córdoba. Los individuos incluidos en el estudio fueron encuestados con el fin de obtener datos clínicos y epidemiológicos como edad, sexo y antecedentes hospitalarios, entre otros. En el caso de los niños, se lo logró la autorización para participar en el estudio a través de un consentimiento informado firmado por los padres de familia. Se excluyeron aquellos individuos que habían sido hospitalizados (hospitales y/o clínicas) en los últimos 6 meses previos a la toma de la muestra. 


\section{Aspectos éticos}

El estudio estuvo clasificado como de riesgo mínimo según la Resolución 8430/93 del Ministerio de Salud y fue aprobado por el comité de ética del Instituto de Investigaciones Biológicas del Trópico. De toda la población estudiada se obtuvo consentimiento escrito de los participantes, en el caso de los menores de edad la autorización fue dada por los padres.

\section{Identificación microbiológica}

Las muestras fueron inoculadas en agar base tripticasa de soya con sangre de cordero al 5\% (v/v) y se incubaron a $37^{\circ} \mathrm{C}$ por 24 horas, aquellas colonias características de Staphylococcus aureus se probaron bioquímicamente mediante pruebas convencionales como catalasa, coagulasa, DNAsa y crecimiento en salado manitol. Como control se utilizó la cepa de referencia ATCC 25923 especie especifica de $S$. aureus.

Los aislamientos que resultaron coagulasa positiva se confirmaron a través de pruebas bioquímicas del sistema MicroScan ${ }^{\mathrm{TM}}$ combo 1A (SIEMENS) que a la vez determina la sensibilidad antimicrobiana para los diferentes antibióticos. Luego, los aislamientos de S. aureus previamente identificados, fueron congelados por triplicado a $-70^{\circ} \mathrm{C}$ en skim milk al $10 \%(\mathrm{p} / \mathrm{v})$, para la realización posterior de los ensayos moleculares.

\section{Identificación de genes de virulencia nuc, mecA y PVL}

Para la detección de los genes $n u c, m e c A$ y PVL se utilizaron los protocolos de Brakstad O, et al (5) y Oliveira D. et al (6) y Gerard L et al (7) respectivamente (Tabla 1).

\section{Análisis de los resultados}

Se utilizó la estadística descriptiva para este tipo de estudios.

\section{Resultados}

De las 253 muestras analizadas, $62(24,5 \%)$ resultaron positivas para Staphylococcus aureus, de éstas 4 (6,45\%) fueron resistentes a meticilina (SARM-AC) de las cuales 2 (25\%) resultaron PVL positivas; 58 (93,54\%) fueron sensibles a meticilina (SASM), 6 (75\%) positivas para PVL, para un total de 8 muestras PVL positivas (Tabla 2).

Tabla 1. Iniciadores utilizados para la detección de los genes nuc, mecA y PVL.

\begin{tabular}{llll}
\hline Gen & Controles & \multicolumn{1}{c}{ Secuencia } & Ref. \\
\hline nuc & ATCC 29213 & Primer1, GCGATTGATGGTGATACGGTT & $(5)$ \\
& & Primer2, AGCCAAGCCTTGACGAACTAAAGC & \\
\hline$m e c A$ & MECA P4, TCCAGATTACAACTTCACCAGG & $(6)$ \\
& Chileno & MECA P7, CCACTTCATATCTTGTAACG & \\
\hline PVL & ATCC 25923 & $l u k-P V-1$, ATCATTAGGTAAAATGTCTGGACATGATCCA & $(7)$ \\
& & $L u k-P V-2$, GCATCAASTGTATTGGATAGCAAAAGC & \\
\hline
\end{tabular}

Tabla 2. Distribución de los aislamientos de SARM y SASM PVL positivos.

\begin{tabular}{lcccc}
\hline S. aureus & $\begin{array}{c}\text { Resistentes a } \\
\text { Meticilina } \\
(\boldsymbol{m e c A})\end{array}$ & $\begin{array}{c}\text { Sensibles } \\
\text { a Meticilina }\end{array}$ & nuc & PVL \\
\hline $\mathbf{n}(\boldsymbol{\%})$ & $\mathbf{n}(\boldsymbol{\%})$ & $\mathbf{n}(\boldsymbol{\%})$ & $\mathbf{n}(\boldsymbol{\%})$ & $\mathbf{n}(\boldsymbol{\%})$ \\
\hline $62(24,5)$ & $4(6,45)$ & $58(93,54)$ & $62(100)$ & $8(12,9)$ \\
\hline
\end{tabular}


El 100\% de las cepas SARM-AC productoras de PVL se aislaron de individuos sanos.

Las 2 cepas de SARM productoras de PVL se aislaron en individuos con edades de 7 y 22 años, de sexo masculino y femenino respectivamente. La prevalencia de las cepas SARM en los internos fue de $1,1 \%$, en los estudiantes universitarios de $1 \%$ y en los niños de 3,23\% (Tabla 3).

La susceptibilidad antimicrobiana de las cepas de SARMAC demostró sensibilidad a eritromicina, ciprofloxacina, rifampicina, cloranfenicol, gentamicina, synercid ${ }^{\mathrm{TM}}$, trimetoprim/sulfametoxazol y vancomicina, además la cepa aislada de los niños fue sensible a estos antibióticos y a clindamicina.

\section{Discusión}

S. aureus resistente a meticilina es un microorganismo emergente que se puede transmitir fácilmente y producir patologías de difícil manejo. Se han descrito factores de riesgo para la adquisición de SARM en la comunidad (SARM-AC) entre los que se encuentra la hospitalización reciente, exposición a antibióticos, enfermedades crónicas, contacto con personas con factores de riesgo, condiciones de hacinamiento y prácticas de higiene inadecuadas, entre otros (8).

La mayoría de los estudios basan la búsqueda de la colonización por SARM en fosas nasales o en la nasofaringe, sin embargo, estudios como el de Lautenbach et al (9) destacaron la importancia de la colonización por SARM en otras sitios anatómicos y aunque la colonización nasal es mayor, también es importante la colonización a nivel de faringe que es el segundo sitio anatómico más colonizado por SARM y que por su ubicación anatómica podría más fácilmente producir infección.

En Colombia son pocos los casos que se han descrito de SARM-AC, Álvarez et al (10), reportó dos casos de pacientes con infecciones de tejidos blandos cuyo agente causal fue SARM productor de PVL, ellos determinaron el origen comunitario con el cassette cromosomal tipo IV. Fortes et al (11) en Brasil, reportó el caso de un paciente previamente sano, con endocarditis por SARM-AC secundaria a una infección por herida traumática, determinando también la presencia de los genes PVL y cassette cromosomal tipo IV. Estos hallazgos resaltan la importancia de la determinación de los genes PVL y cassette cromosomal tipo IV (12).

Sin embargo, existen estudios recientes que han generado controversia acerca del papel de PVL en la patogenicidad de la infección por SARM-AC. Voyich et al (13) argumentan que PVL no es necesario para la patogénesis de $S$. aureus en sepsis o bacteriemia ni en abscesos de piel, en contraste, Labandeira et al (14) sugieren que PVL es suficiente para causar neumonía y que la expresión de la leucotoxina induce cambios transcripcionales en los genes que terminan secretando la proteína inflamatoria pulmonar Spa. Wardenburg et al (15) afirman que PVL no sólo no es necesaria para la patogénesis de la infección por $S$. aureus sino que sugiere que esta toxina puede preparar la respuesta inmune del huésped, promoviendo una respuesta inflamatoria que facilita el reconocimiento del patógeno. Diep et al (16) reportó que PVL es importante en las primeras 24-48 horas de la infección, pero que posteriormente el papel de la PVL se transforma en modesto y no contribuye al deterioro inmune.

En nuestra investigación se aislaron cepas SARM productoras de PVL, pero no se identificó el tipo de cassette. En este sentido, aunque nuestro trabajo carece de la detección molecular de los cassettes, con base en los perfiles de susceptibilidad antimicrobiana de estos aislamientos, la producción de la PVL y sus características epidemiológicas, se podría decir que estos cepas son compatibles con SARM-AC, que a diferencia de las cepas de SARM-AH, son sensibles a otros antibióticos no $\beta$-lactámicos (17). Aunque estudios recientes demuestran

Tabla 3. Distribución de SARM en los tres grupos estudiados.

\begin{tabular}{lccc}
\hline & \multicolumn{3}{c}{ Cepas SARM } \\
\cline { 2 - 4 } GRUPO & $\mathbf{n}$ & $\mathbf{n}$ & $\mathbf{\%}$ \\
\hline Internos & 91 & 1 & 1,1 \\
Estudiantes universitarios & 100 & 1 & 1 \\
Niños en edad escolar & 62 & 2 & 3,23 \\
TOTAL & 253 & 4 & 5,33 \\
\hline
\end{tabular}


la presencia el cassette cromosomal tipo IV en cepas de SARM-AH, y discuten que con el tiempo éste no sería un indicador adecuado para determinar el origen comunitario (17-19).

Otro estudios como el de McMullen et al (20) tuvieron en cuenta además la sensibilidad a clindamicina y trimetoprin/sulfametoxazol, para clasificar los aislamientos como adquiridos en la comunidad. En nuestro trabajo todas las cepas fueron sensibles a eritromicina, quinolonas (ciprofloxacina) y trimetoprin/sulfametoxazol. La cepa aislada de los niños fue además sensible a clindamicina, lo que sugiere el origen comunitario si se tiene en cuenta que los SARM-AC son susceptibles frecuentemente a antibióticos no ß-lactámicos (2).

El hallazgo de pacientes sanos portadores de SARM productor de PVL, en nariz o faringe, genera preocupación en salud pública, por la forma como circulan estas cepas en la comunidad, esto fue descrito por Hernández et al (21), quien realizó un estudio en Cuba para la detección de portadores nasales de SARM entre niños hospitalizados y niños sanos de la comunidad, encontrando que un $0,35 \%$ de los niños sanos eran portadores, los resultados de nuestra investigación muestra una mayor prevalencia, con un 3,23\% de niños en edad preescolar son portadores de SARM productor de PVL a nivel de faringe.

Por otro lado, Mertz et al (22) investigó la presencia de $S$. aureus en una población de 3464 individuos adultos jóvenes, de los cuales $12,4 \%$ fueron portadores a nivel de faringe, los cuales no presentaban ninguno de los factores de riesgo para adquirir estas cepas, lo que podría constituir a esta población como de alto riesgo para portar SARMAC y así diseminarlo, de igual forma en nuestro estudio también se encontró a un joven portador de SARM productor de PVL.

SARM ha producido en varias oportunidades brotes importantes en internos de cárcel en varias ciudades en Estados Unidos y Canadá (23-27). Tattevin et al (28) por su parte describe un estudio retrospectivo desde el año 2001 al 2007 realizado en la cárcel de San francisco donde hubo un marcado predominio del clon USA300. Lowy et al (29) realizo un estudio sobre la prevalencia de la colonización nasal de SARM en internos de 2 cárceles del estado de Nueva York encontrando que el 25,5\% estaban colonizados por $S$. aureus de los cuales el 10,5\% eran SARM y de estos el $61,5 \%$ eran productores de PVL. En nuestro estudio la prevalencia entre los internos de la cárcel a nivel de faringe fue sorprendentemente de apenas el $1,1 \%$, probablemente debido al tamaño más pequeño de la población estudiada.
También se ha analizado la manera como se pueden diseminar estas cepas entre individuos con contacto cerrado, como integrantes de una misma familia o jardines infantiles, donde se han hallado individuos portadores de SARM-AC, tal es el caso de un estudio realizado en Copenhague, Dinamarca (30) donde se estudiaron 16 familias de las cuales 7 eran portadoras de SARM-AC. En este estudio se determino que la transmisión ocurrió mediante el contacto entre una persona portadoras y no portadoras, igualmente en nuestro estudio se encontraron portadores de SARM$\mathrm{AC}$ en un centro de cuidado infantil lo que demuestra la susceptibilidad de esta población a portar y transmitir este microorganismo.

Al comparar los tres grupos estudiados se aprecia una mayor prevalencia de cepas SARM-AC en los niños en edad escolar, que en los adultos jóvenes y los internos (Tabla 3), lo que demuestra que los niños son más susceptibles a esta colonización debido a las actividades que ellos realizan y el contacto cercano con los demás niños.

A pesar de la baja prevalencia de SARM-AC, es preocupante el hallazgo en niños que no presentan ningún tipo de manifestación clínica, por lo que se recomienda estimularlos a tener prácticas de higiene adecuadas como lavarse las manos frecuentemente y no compartir los elementos de aseo personal con el fin de reducir el riesgo de infecciones y la diseminación de estas cepas.

Es preocupante lo común que se esta volviendo encontrar cepas SARM-AC en ambientes nosocomiales y cepas hospitalarias en la comunidad $(18,31)$, de hecho ya existe un solapamiento clonal y pronto la utilidad de la PFGE y los clones quedaría sin valor epidemiológico.

\section{Conclusión}

Los resultados de este trabajo, hasta ahora el primero realizado en Córdoba-Colombia, demuestran la presencia de individuos sanos colonizados por cepas de SARM productoras de PVL y con características fenotípicas de cepas de SARM-AC, las cuales se aislaron de niños en edad escolar y adultos jóvenes, sin exposición previa a centros hospitalarios o factores de riesgo para la adquisición de estas cepas. El posible riesgo que existe con el portador sano, actuando como un diseminador de estas cepas a otras personas de la comunidad, genera preocupación en salud publica ya que representa un riesgo de transmisión y la probabilidad de brotes por SARM-AC. 


\section{Agradecimientos}

Los resultados parciales de este articulo hacen parte del trabajo de grado de Magister en Microbiología tropical de D. Lozano. A los doctores Jinneth Reyes y Cesar Arias por facilitarnos las cepas controles de S. aureus.

\section{Financiación}

Este trabajo fue financiado por el Centro de Investigaciones de la Universidad de Córdoba (CIUC), código FMV01-07.

\section{Conflicto de intereses}

Los autores declaran que no existen conflictos de intereses.

\section{Referencias}

1. Boyle-Vavra S, Daum R. Community-acquired meticilin-resistant Staphylococcus aureus: the role of Panton-Valentine leukocidin. Laboratory Investigation. 2007; 87: 3-9.

2. Borraz C. Epidemiología de la resistencia a meticilina en cepas de Staphylococcus aureus aisladas en hospitales españoles. Tesis doctoral. Universidad de Barcelona. Barcelona. 2006; p 8.

3. Maltezou H, Giamarellou H. Community-acquired methicillin-resistant Staphylococcus aureus infections. International Journal of Antimicrobial Agents. 2006; 27:87-96.

4. Cortes J, Gomez C, Cuervo S, Leal A. Implicaciones en salud pública de Staphylococcus aureus Meticilino resistente adquirido en la comunidad en Bogotá, Colombia. Revista de Salud Publica. 2007; 9 (3): 448-454.

5. Brakstad O, Aabakk K y Maeland J. Detection of Staphylococcus aureus by polymerasa chain reaction amplification of the nuc gene. Journal of Clinical Microbiology. 1992; 30: 1654-1660.

6. Oliveira D, De Lencastre H. Multiplex PCR estrategy for rapid identification of structural types and variands of the mec element in meticilin-resistan Staphylococcus aureus. Antimicrobial Agents and Chemotherapy. 2002; 46: 2155-2161.

7. Gerard L, Yves Pie M, Florence G, Michele B, MarieOdile P, Valerie G, Francois V, Jerome E. Involvement of Panton-Valentine Leukocidin-producing Staphylococcus aureus in primary skin infections and pneumonia. Clinical Infectious Diseases. 1999; 29: 1128-1132.

8. Fritz S, Garbutt J, Elward A, Shannon W, Storch G. Prevalence of and risk factors for community-acquired methicillin-resistant and methicillin-sensitive staphylococcus aureus colonization in children seen in a practice-based research network. Pediatrics. 2008; 121 (6):1090-1098.

9. Lautenbach E. Et Al. Surveillance Cultures for Detection of Methicillin- Resistant Staphylococcus aureus: Diagnostic Yield of Anatomic Sites and Comparison of Providerand Patient-Collected Samples Infection Control and Hospital Epidemiology 2009; 30 (4): 380-382

10. Alvarez C, Barrientes O, Leal A, Contreras G, Barrero L, Rincón S, Diaz L, Vanegas N, Arias C. Community associted methicillin resistant $S$. aureus, Colombia. Emerging Infectious Disesases. 2006; 12: 20002001.

11. Fortes C, Espanha C, Bustorff F, Zappa B, Ferreira A, Moreira R, Pereira N, Fowler V, Deshmukh H. First reported case of infective endocarditis caused by community-acquired methicillin-resistant Staphylococcus aureus not associated with healthcare contact in Brazil. Brazilian Journal of Infection Diseases. 2008;12: 541-543.

12. Kefala-Agoropoulou K, Protonotariou E, Vitti D, Sarafidou S, Anastasiou A, Kollios K, Roilides E. Lifethreatening infection due to community-acquired methicillin-resistant Staphylococcus aureus: case report and review. European Journal of Pediatric. $2010 ; 169$ (1):47-53.

13. Voyich JM, Otto M, Mathema B, et al. Is Panton-Valentine leukocidin the major virulence determinant in community-associated methicillin resistant Staphylococcus aureus disease? Journal Infection diseases 2006; 194:1761-1770.

14. Labandeira-Rey M, Couzon F, Boisset S, et al. Staphylococcus aureus Panton-Valentine leukocidin causes necrotizing pneumonia. Science 2007; 315: $1130-1133$.

15. Wardenburg J, Palazzolo-Ballance A, Otto M, Schneewind $\mathrm{O}$ y DeLeo F. Panton-Valentine Leukocidin Is Not a Virulence Determinant in Murine Models of Community-Associated Methicillin-Resistant Staphylococcus aureus Disease. Journal Infection diseases 2008;198: 1166-1170 
16. Diep BA, Palazzolo-Ballance AM, Tattevin P, Basuino L, Braughton KR, Whitney AR, Chen L, Kreiswirth BN, Otto M, DeLeo FR, Chambers HF. Contribution of Panton-Valentine leukocidin in communityassociated methicillin-resistant Staphylococcus aureus pathogenesis. PLoS One 2008; 3: e3198.

17. Jung S, Shin D, Park K, Shin J. Antimicrobial susceptibility and clonal relatedness between community- and hospital-acquired methicillinresistant Staphylococcus aureus from blood cultures. Journal of Microbiology. 2006; 44(3):336-343.

18. Miller L, Kaplan S. Staphylococcus aureus: A Community Pathogen. Infectious Diseases Clinical of North America. 2009; 23: 35-52.

19. Yao D Et. Al. Molecular characterization of Staphylococcus aureus isolates causing skin and soft tissue infections (SSTIs). BMC Infectious Diseases 2010, 10(1): 133

20. McMullen K, Warren D, Woeltje K. The changing susceptibilities of methicillin-resistant Staphylococcus aureus at a midwestern hospital: the emergence of "community-associated" MRSA. American Journal of Infection and Control. 2009; 3:1-4.

21. Hernández I, Toraño G, González M, González I. Staphylococcus aureus resistente a la meticilina: detección de portadores entre niños hospitalizados y niños sanos de la comunidad. Revista Cubana de Medicina Tropical 2003; 55: 153-161.

22. Mertz D, Frei R, Periat N, Zimmerli M, Battegay M, Fl?ckinger U, Widmer AF. Exclusive Staphylococcus aureus throat carriage: at risk populations. Archives of Internal Medicine. 2009; 169:172 -178.

23. Baillargeon J, Kelley M, Leach C, Baillargeon G, y Pollock B. Methicillin-resistant Staphylococcus aureus infection in the Texas prison system. Clinical Infectious Diseases 2004; 38:92-95.

24. Centers for Disease Control and Prevention. Methicillin-resistant Staphylococcus aureus infections in correctional facilities-Georgia, California, and Texas, 2001-2003. MMWR 2003; 52:992-996.
25. Centers for Disease Control and Prevention. Methicillin-resistant Staphylococcus aureus skin or soft tissue infections in a state prison-Mississippi, 2000. MMWR 2001; 50:919-922.

26. Main C, Jayaratne P, Haley A, Rutherford C, Smaill F, y Fisman D. Outbreaks of infection caused by community-acquired methicillin- resistant Staphylococcus aureus in a Canadian correctional facility. Canadian Journal Infection Diseases and Medical Microbiology 2005; 16:343-348.

27. Pan, E. S., B. A. Diep, H. A. Carleton, E. D. Charlebois, G. F. Sensabaugh, B. L. Haller, and F. PerdreauRemington.. Increasing prevalence of methicillin-resistant Staphylococcus aureus infection in California jails. Clinical Infectious Diseases 2003; 37:13841388 .

28. Tattevin P, An Diep B, Jula M y Perdreau-Remington F. Long-Term Follow-Up of Methicillin-Resistant Staphylococcus aureus Molecular Epidemiology after Emergence of Clone USA300 in San Francisco Jail Populations. Journal of Clinical Microbiology, 2008; 46(12):4056-4057.

29. Lowy, F. D., A. E. Aiello, M. Bhat, V. D. JohnsonLawrence, M. H. Lee, E. Burrell, L. N. Wright, G. Vasquez, and E. L. Larson.. Staphylococcus aureus colonization and infection in New York state prisons. Journal of Infection Diseases 2007;196: 911-918.

30. Bartels M, Kristoffersen K, Boye K, Westh H. Rise and subsequent of community associate methicillin resistant Staphylococcus aureus ST30- IVc in Copenhagen, Denmark through and effective search and destroy policy. Clinical Microbiology Infection. 2010; 16 (1): 78-83.

31. Campana S, Cocchi P, Döring G, Taccetti G, Moroney SM. Emergence of an epidemic clone of communityassociated methicillin-resistant panton-valentine leucocidin-negative Staphylococcus aureus in cystic fibrosis patient populations. Journal of Clinical Microbiology 2007; 45: 3146-3147. 